\title{
SEMI-ORTHOGONAL USER SELECTION FOR MISO SYSTEMS WITH QUANTIZED FEEDBACK
}

Hajer KHANFIR , Didier LE RUYET

Electronics and Communications Laboratory, CNAM, 292 rue Saint Martin, 75141, Paris, France

khiari, leruyet @enam.fr
Berna ÖZBEK

Electrical and Electronics Engineering Department, Izmir Institute of Technology, Urla, 35430, Izmir,Turkey

bernaozbek@iyte.edu.tr

\begin{abstract}
For MISO multi-user downlink wireless communication system with precoding at the transmission, the channel state information at the transmitter can provide tremendous capacity gains. However, the amount of feedback data increases with the number of users in the cell and the number of transmit antennas. In this paper, we study on different algorithms and criteria in order to significantly reduce the amount of feedback data. We associate the classical norm criterion with a criterion based on the orthogonality between the user channels. Without cooperation between the users, we only allow users that are semi-orthogonal to feedback their channel information (CQI and CDI) to the base station. The feedback CDI is quantized using a local grassmanian packing. We show that the proposed combined criterion with a finite feedback rate gives better performance compared to the norm criterion. Furthermore we show that the performance is not affected by CQI quantization. ${ }^{12}$
\end{abstract}

\section{INTRODUCTION}

In a multi-user downlink system, it is possible to increase the total throughput by using $N_{t}$ multiple transmit antennas at the base station. To achieve a higher data rate, the base station must transmit to more than one user simultaneously, and exploit spatial diversity offered by multiple transmit antennas, by means of Space Division Multiple Access (SDMA). When $N_{t}<K$, the architecture of a multi-user multi-antennas system requires not only a sophisticated precoding scheme but also an efficient user selection algorithm to reduce the feedback load [1]. The selected users must send their channel quality information (CQI) and channel direction information (CDI) for the reduction of interference. In fact the transmitter aided by the feedback information can improve the sum-rate of the system through the right precoding and selected users. In this paper, we propose to reduce the feedback information

\footnotetext{
${ }^{1}$ The work of Hajer Khanfir and Didier Le Ruyet was supported by the Euripides European project SMART.

${ }^{2}$ The work of Berna Özbek was supported by the FP6-IYTE wireless project.
}

thanks to the selection of a group of semi-orthogonal users at the user side. In order to limit the feedback rate, the CDI will be quantized using a local Grassmannian packing. Finally, we will evaluate the impact of the quantization on system performance.

\section{SYSTEM MODEL}

Let $N_{t}$ be the number of antennas at the transmitter, and consider a cluster of $K$ mobile users, each equipped with a single receive antenna. The received signal is corrupted by Additive White Gaussian Noise (AWGN). We suppose that all the users are independent and distributed in a homogeneous way: they are at the same distance of the BS and the average signal-tonoise ratio (SNR) is the same for all the receivers.

At every block, the signal at the $k$-th user, $k=1,2, \ldots . K$, can be written as:

$$
y_{k}=\mathbf{h}_{k} \mathbf{x}+n_{k} \quad k=1, \cdots, K
$$

where $\mathbf{x}=\mathbf{W P s}=\sum_{k=1}^{K} \sqrt{P_{k}} \mathbf{w}_{k} s_{k}$, where $\mathbf{P}=$ $\operatorname{diag}\left(\sqrt{P_{1}}, \cdots, \sqrt{P_{K}}\right)$, the matrix for power loading, $\mathbf{W}=$ $\left[\mathbf{w}_{1} \cdots \mathbf{w}_{K}\right]$ is a precoding matrix (the columns of $\mathbf{W}$ are thus normalized to unit norm), $\mathbf{x} \in \mathbb{C}^{N_{t} \times 1}$ is the transmitted symbol from the base station antennas, subject to a short-term power constraint ( the transmitted signal must satisfy power constraint $\mathbf{x}^{H} \mathbf{x} \leq P$ ) and we consider equal power allocation over each transmit beam, $\mathbf{h}_{k} \in \mathbb{C}^{1 \times N_{t}}$ is the channel gain vector to the $k$-th user with i.i.d (independently and identically distributed ) complex Gaussian entries and $\mathrm{s}$ is the transmitted data for the scheduled users at time $T$. The complex coefficients of the channel vector and noise $n_{k} \in \mathbb{C}$ are i.i.d and zero mean gaussian variables. We normalize the channel such that the entries of $\mathbf{h}_{k}$ have unit variance.

Equation (1) can also be written in matrix form as following :

$$
\mathbf{y}=\mathbf{H x}+\mathbf{n}
$$

where $\mathbf{y}=\left[y_{1}, \ldots, y_{K}\right]^{T}$ is the received vector, $\mathbf{n}=$ 
$\left[n_{1}, \ldots, n_{K}\right]^{T}$ is the noise vector and $\mathbf{H}$ is the $K \times N_{t}$ channel matrix.

Using frequency division duplexing (FDD) system, we assume that the channel is perfectly known at the receiver through, e.g. pilot-assisted training, but not at the transmitter. In this paper, for simplicity we consider that the feedback channels are not affected by delay and are error-free .

\section{PRECODING STRATEGY}

For a system where $N_{t}<K$, let $\mathcal{S}$ be the set of $N_{t}$ users scheduled at time $T$. Then the associated users'data are transmitted via Zero-forcing Beamforming (ZFBF) precoding [2] by exploiting the channel state information at the transmitter $(\mathrm{CSIT})$. Then $\mathbf{H}(\mathcal{S})$ denotes the matrix consisting of $N_{t}$ channel vectors of the selected users at time $T$. The relation between the data vector $\mathbf{s}(\mathcal{S})$ and the transmitted vector $\mathbf{x}(\mathcal{S})$ is given by:

$$
\mathbf{x}(\mathcal{S})=\mathbf{W}(\mathcal{S}) \mathbf{P}(\mathcal{S}) \mathbf{s}(\mathcal{S})
$$

where $\mathbf{P}=\operatorname{diag}\left(\sqrt{\frac{P}{N_{t}}}, \cdots, \sqrt{\frac{P}{N_{t}}}\right)$, is the $N_{t} \times N_{t}$ matrix for uniform power loading. The $\mathrm{ZF}$ transmit beamforming vector is : $\mathbf{W}(\mathcal{S})=\alpha \mathbf{H}(\mathcal{S})^{H}\left(\mathbf{H}(\mathcal{S}) \mathbf{H}(\mathcal{S})^{H}\right)^{-1}$

In order to keep the short term power constant we have :

$$
\alpha=\frac{1}{\sqrt{\operatorname{tr}\left(\left(\mathbf{H}(\mathcal{S}) \mathbf{H}(\mathcal{S})^{H}\right)^{-1}\right)}}
$$

The sum rate achieved by the ZFBF scheme is :

$$
R_{Z F B F}(\mathcal{S})=\max _{\mathbf{w}_{k}} \sum_{k \in \mathcal{S}} \log \left(1+S I N R_{k}\right)
$$

The signal-to-interference-plus-noise-ratio (SINR) for every user is :

$$
S I N R_{k}=\frac{\left|\mathbf{h}_{k} \mathbf{w}_{k}\right|^{2}}{\sigma^{2}+\sum_{j \neq k}^{N_{t}}\left|\mathbf{h}_{k} \mathbf{w}_{j}\right|^{2}}
$$

The achievable sum rate of $\mathrm{ZFBF}$ is found by considering every possible choice of user groups $\mathcal{S}$ :

$$
R_{Z F B F}=\max _{\mathcal{S} \subset\{1, \cdots, K\}:|\mathcal{S}|=N_{t}} R_{Z F B F}(\mathcal{S})
$$

\section{USER SELECTION CRITERIA}

To maximize the sum rate of the downlink system under an average power constraint $P$, it is first of all necessary to choose the best combination of $N_{t}$ users. The exhaustive search which consists in evaluating $\left(\begin{array}{c}K \\ N_{t}\end{array}\right)$ combinations quickly becomes prohibitive. However, the users having a poor channel (low norm or/and interfering with other good users) should not take part in the user selection algorithm, nor feedback their channel information.

\subsection{Construction of selected user group}

In order to select the users we propose three self discrimination criteria. Let us consider the following sets:

The norm-only criterion only selects users whose channel norms are above a threshold $\gamma_{t h}[1]$.

$$
\mathcal{T}_{1}=\left\{k \in \mathcal{R}:\left\|\mathbf{h}_{k}\right\|^{2}>\gamma_{t h}\right\}
$$

Where $\mathcal{R}$ be the set of all users.

$\mathcal{T}_{2}:$ the set of near orthogonal users.

The semi-orthogonal criterion selects users whose channel direction $(\mathrm{CDI})$ are semi-orthogonals. Each user generates the same $N_{t}$ random orthonormal vectors $\phi_{i}\left(N_{t} \times 1\right), i=1, \ldots, N_{t}$. The users measure the orthogonality between their channels and the random vectors $\phi_{i}$ using the chordal distance:

$$
d^{2}\left(\overline{\mathbf{h}}_{k}, \phi_{i}\right)=1-\left|\overline{\mathbf{h}}_{k}^{H} \phi_{i}\right|^{2}
$$

where $\overline{\mathbf{h}}_{k}=\frac{\mathbf{h}_{k}}{\left\|\mathbf{h}_{k}\right\|}$ is the normalized channel vector of the user $k$.

Let $\mathbb{O}^{N_{t}}$ be the unit sphere lying in $\mathbb{C}^{N_{t}}$ and centered at the original. Using the chordal distance metric, for any $\epsilon_{t h}<$ 1 , we can define a spherical cap on $\mathbb{O}^{N_{t}}$ with center $\mathbf{o}$ and square radius $\epsilon_{t h}$ as the open set :

$$
\mathcal{B}_{\epsilon}(\mathbf{o})=\left\{\mathbf{g} \in \mathbb{O}^{N_{t}}: d^{2}(\mathbf{g}, \mathbf{o}) \leq \epsilon_{t h}\right\}
$$

For the second criterion each user generates a set $\mathcal{F}=$ $\left\{\phi_{1}, \ldots, \phi_{N t}\right\}$ composed of $N_{t}$ unitary orthogonal vectors.

Then we have:

$$
\mathcal{T}_{2}=\left\{k \in \mathcal{R}: \overline{\mathbf{h}}_{k} \in \bigcup_{i=1}^{N_{t}} \mathcal{B}_{\epsilon}\left(\phi_{i}\right)\right\}
$$

The last criterion combines the two previous ones:

$$
\mathcal{T}_{3}=\left\{k \in \mathcal{R}: \overline{\mathbf{h}}_{k} \in \bigcup_{i=1}^{N_{t}} \mathcal{B}_{\epsilon}\left(\phi_{i}\right) \text { and }\left\|\mathbf{h}_{k}\right\|^{2}>\gamma_{t h}\right\}
$$

\subsection{Thresholds predetermination values}

We must choose the threshold values for the three sets in order to allow the number of average users $K_{\text {avg }}$ to feedback their CDI and CQI.

The channel norm and its direction are independent and we have:

$$
\begin{aligned}
K_{\text {avg }} & =K \mathcal{P}\left\{k \in \mathcal{T}_{3}\right\} \\
& =K \mathcal{P}\left\{k \in \mathcal{T}_{1}\right\} \times \mathcal{P}\left\{k \in \mathcal{T}_{2}\right\} \\
& =K \mathcal{P}\left\{\left\|\mathbf{h}_{k}\right\|^{2}>\gamma_{t h}\right\} \times \mathcal{P}\left\{k \in \mathcal{R}: \overline{\mathbf{h}}_{k} \in \bigcup_{i=1}^{N_{t}} \mathcal{B}_{\epsilon}\left(\phi_{i}\right)\right\}
\end{aligned}
$$




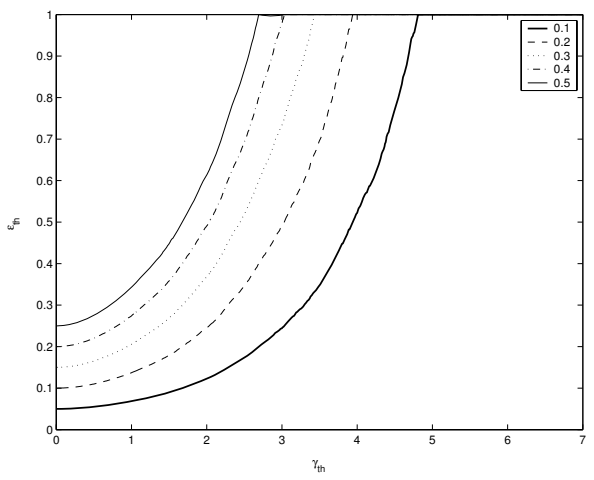

Fig. 1. $\epsilon_{t h}$ and $\gamma_{t h}$ for $\mathcal{P}\left\{k \in \mathcal{T}_{3}\right\}=0.1$ to 0.5

According to [3]

$$
\mathcal{P}\left\{k \in \mathcal{R}: \overline{\mathbf{h}}_{k} \in \bigcup_{i=1}^{N_{t}} \mathcal{B}_{\epsilon}\left(\phi_{i}\right)\right\}=\epsilon^{N_{t}-1}
$$

Using the union bound theorem:

$$
K_{\text {avg }} \leq K N_{t} \quad \mathcal{P}\left\{\left\|\mathbf{h}_{k}\right\|^{2}>\gamma_{t h}\right\} \epsilon^{N_{t}-1}
$$

The set $\mathcal{T}_{1}$ is determined by the incomplete gamma distribution $\operatorname{Gamma}\left(N_{t}, 1\right)$ which can be bounded by [4][5]:

$$
\left[1-e^{-\beta \gamma}\right]^{N_{t}} \leq \int_{0}^{\gamma_{t h}} f_{\gamma}(\gamma) d \gamma \leq\left[1-e^{-\gamma}\right]^{N_{t}}
$$

where $\beta=\left(N_{t} !\right)^{-\frac{1}{N_{t}}}$ and $f_{\gamma}(\gamma)$ is the probability density function $\chi_{2}^{2}\left(N_{t}\right)$.

In Fig. 1 we present the curves of the pair $\left(\gamma_{t h}, \epsilon_{t h}\right)$ allowing predetermined probability $\mathcal{P}\left\{k \in \mathcal{T}_{3}\right\}$ (10\% to $\left.50 \%\right)$. For each probability, it will be necessary to choose a pair by privileging either the criterion on the norm or the criterion on orthogonality.

\section{QUANTIZED FEEDBACK LINK}

To address the lack of perfect CSIT, a classical solution is to quantize CDI and CQI before transmission over the finite rate feedback link. In [6], the Lloyd algorithm was suggested for the design of the beamforming vector codebook. The codebook should be constructed by minimizing the maximum inner product between codewords and this results in the Grassmannian line packing solution when the channel vector is i.i.d.

In the quantized feedback scheme, the precoding vector w is taken from a set of $2^{B}$ vectors where $B$ is the number of feedback bits.
In contrast to the normalized i.i.d. channel isotropically distributed in $\mathbb{O}^{N_{t}}$, an important aspect of a limited feedback codebook tailored to a spherical cap region is the quantization of the localized region or local packing. A local Grassmannian packing with parameters $N_{t}, N, \mathbf{o}, \epsilon_{t h}$ is a set of $N$ vectors, where $N$ is the codebook size, $\mathbf{w}_{i}, i=1, \ldots, N$, constrained to a spherical cap $\mathcal{B}_{\epsilon}(\mathbf{o})$ in $\mathbb{O}^{N_{t}}$ such that

$$
\min _{1 \leq i<j \leq N} d^{2}\left(\mathbf{w}_{i}, \mathbf{w}_{j}\right)
$$

is maximized.

From the spherical cap $\mathcal{B}_{\epsilon}(\mathbf{o})$ it is possible to compute the rotated spherical cap $\mathcal{B}_{\epsilon}\left(\mathbf{o}_{\text {rot }}\right)$ by applying the following rotation map:

$$
r(\mathbf{o})=\mathbf{U}_{r o t} \mathbf{o} \triangleq \mathbf{o}_{r o t}
$$

where $\mathbf{U}_{\text {rot }}$ is the unitary rotation matrix [7].

As in the i.i.d. case, we use vector quantization to design these local packings. For the $\mathcal{T}_{2}$ and $\mathcal{T}_{3}$ criterion, the codebook must be adapted according to the orthogonal vectors $\phi_{i}$. From the local packing, it is possible to compute the local packing associated to a rotation using the rotation matrix. When, the user CDI is inside the spherical cap region, the user will feedback $\log _{2}(N)$ bits corresponding to the codebook index. In addition to that, it will be necessary to feedback $\log _{2}\left(N_{t}\right)$ bits corresponding to the index of the vector $\phi_{i}$. Consequently, for a codebook size $N, B=\log _{2}\left(N \times N_{t}\right)$ bits will be necessary to quantify the CDI.

\section{SIMULATION RESULTS}

We consider $N_{t}=2$ antennas at the base station. $\gamma_{t h}$ for criterion $\mathcal{T}_{1}$ and the pair $\left(\gamma_{t h}, \epsilon_{t h}\right)$ for criterion $\mathcal{T}_{3}$ are theoretically calculated in order to have an average number of users in the cell $K_{a v g}=4$. Only these users feedback their $Q$ bits for the quantization of each channel gain $\left\|\mathbf{h}_{k}\right\|^{2}$ and $B$ bits corresponding to the codebook index of their quantized CDI. Exploiting this feedback information, the base station will select the $N_{t}$ users in order to maximize the sum data rate.

In all our simulations we take the case where $K_{\text {avg }}=$ 4 and so $\gamma_{t h}$ and the pair $\left(\gamma_{t h}, \epsilon_{t h}\right)$ should be chosen such that $\gamma_{t h}=\left[\begin{array}{llllll}0 & 1.7 & 3 & 4 & 5 & 5.8\end{array}\right]$ for criterion $\mathcal{T}_{1}$, and the pair $\left(\gamma_{t h}, \epsilon_{t h}\right)=[(0,1)(1,0.35)(2,0.23)(2.5,0.18)(3,0.1)(3.8,0.09)]$ for criterion $\mathcal{T}_{3}$.

In Fig. 2 and Fig. 3 we compare respectively the sum rate performances of $\mathcal{T}_{1}$ and $\mathcal{T}_{3}$ schemes at $S N R=15 \mathrm{~dB}$, for different number of active users in the cell and number of feedback bits $F=B+Q$ per user. For $F=4,6$ and 8 bits, we modify the number of CDI bits (B) and CQI bits (Q). As shown in these two figures, the performances are almost independent of the number of CQI bits. Surprisingly, the sum-rate performance is not affected when there is no feedback information about the channel norm $(Q=0)$ of the selected users. On the opposite, the number of CDI bits has a huge impact 


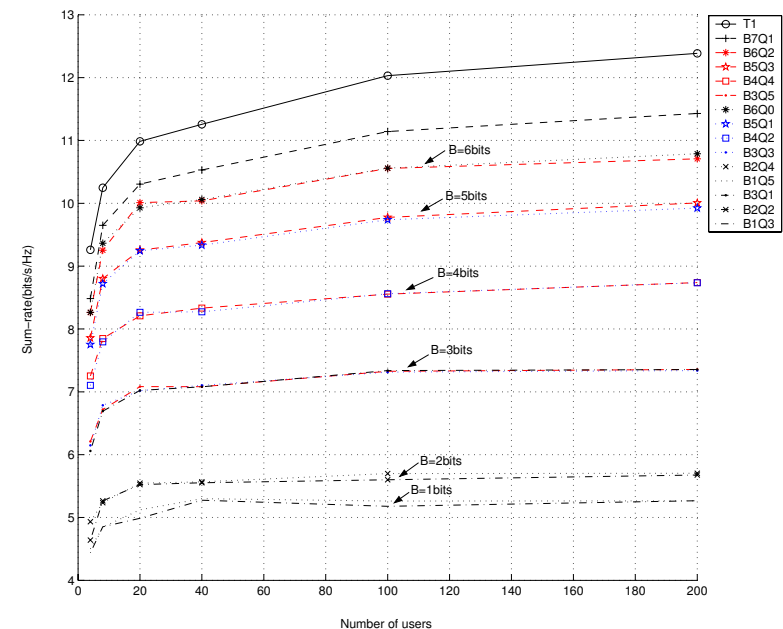

Fig. 2. Sum rate versus the number of users for Criterion $\mathcal{T}_{1}$ under $N_{t}=2, S N R=15 d B, K_{\text {avg }}=4$ users and various feedback bits.

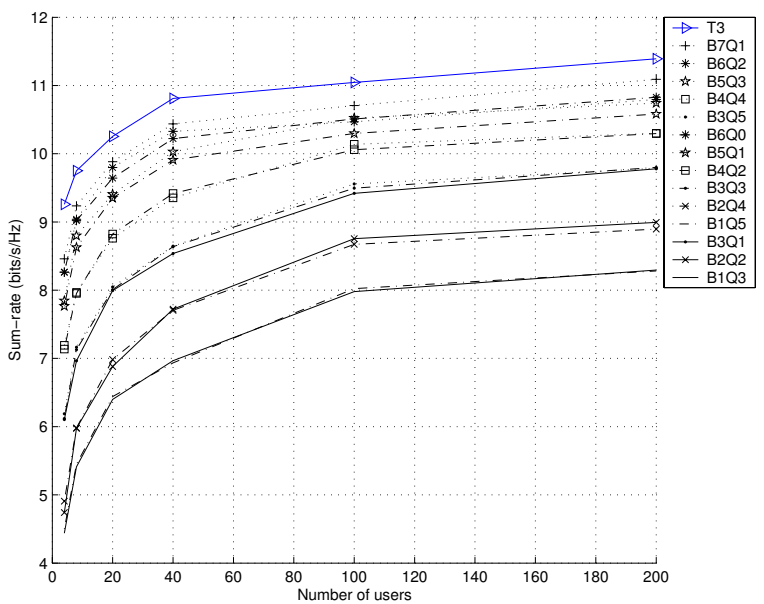

Fig. 3. Sum rate versus the number of users for Criterion $\mathcal{T}_{3}$ under $N_{t}=2, S N R=15 d B, K_{a v g}=4$ users and various feedback bits.

on the performance. For example the performance of the case $B=6 Q=0(F=6)$ are better than the case $B=5 Q=3$ $(F=8)$ even if the former uses less feedback bits than the latter.

In Fig. 4 we compare $\mathcal{T}_{1}$ and $\mathcal{T}_{3}$ performances for different amount of feedback bits for each selected user. As it is shown $\mathcal{T}_{1}$ outperforms $\mathcal{T}_{3}$ in the case of $F=8$ bits, but the two criteria have slightly the same sum-rate performance for $F=6$ feedback bits. For the case of less feedback bits such as $F=4$, not only $\mathcal{T}_{3}$ sum-rate performance becomes better than $\mathcal{T}_{1}$ performance but also the $\mathcal{T}_{1}$ degradation is larger

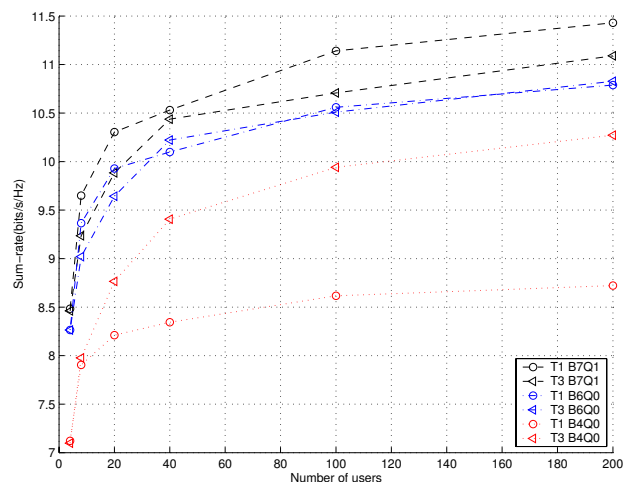

Fig. 4. Sum rate versus the number of users for the Criteria $\mathcal{T}_{1}$ and $\mathcal{T}_{3}$ under $N_{t}=2, S N R=15 d B, K_{\text {avg }}=4$ users and various feedback bits.

relative to $\mathcal{T}_{3}$ loss. This is due to the smaller vector quantization error for $\mathcal{T}_{3}$ criterion since all the codebook vectors are lying in the spherical cap described by the square radius $\left(\epsilon_{t h}\right)$ instead of all the hypersphere.

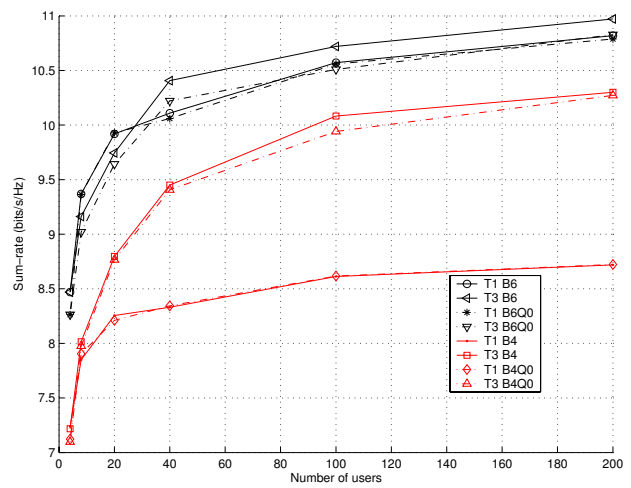

Fig. 5. Sum rate versus the number of users for Criterion $\mathcal{T}_{1}$ and $\mathcal{T}_{3}$ under $N_{t}=2, S N R=15 d B$ and various B and Q.

In Fig. 5 we compare the CQI effects on $\mathcal{T}_{1}$ and $\mathcal{T}_{3}$ sumrate performances. We plot sum-rate performances for various CQI quantization $Q=0$ and $Q=\infty$ (which mean without norm quantization). We confirm the previous results that CQI quantization has no effect on $\mathcal{T}_{1}$ criterion. For $\mathcal{T}_{3}$ criterion the CQI quantization has slight impact on performance with a high resolution.

In Fig. 6 we present the sum-rate versus average SNR for the system with $K=100, F=4,8$ bits such as $B=7$ $Q=1$ and $B=4 Q=0$. We also plot RBF, $\mathcal{T}_{1}$ and $\mathcal{T}_{3}$ schemes without quantization. It is known that RBF performs in a system with large number of users with requirement of all users channel feedback. However, in the case of $F=8$ bits $\mathcal{T}_{1}$ and $\mathcal{T}_{3}$ outperforms RBF scheme with reduced feedback. 


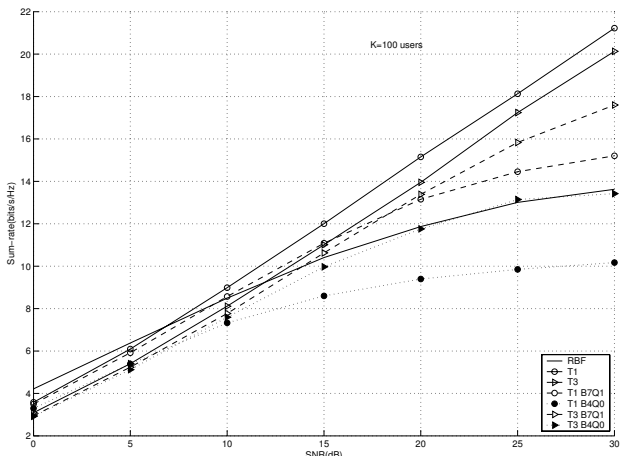

Fig. 6. Sum rate versus snr for RBF, Criterion $\mathcal{T}_{1}$ and $\mathcal{T}_{3}$ under $N_{t}=2, K=100$ and 4,8 bits.

$\mathcal{T}_{3}$ criterion outperforms $\mathcal{T}_{1}$ criterion at high SNR where the system becomes sensitive to the interference and the users's orthogonality is more important. For the case of $F=4$ bits the performance of $\mathcal{T}_{3}$ is almost the same as $\mathcal{T}_{1}$ at low SNR and the same as RBF scheme at high SNR. In this case $\mathcal{T}_{1}$ degradation becomes rapidly compared to the $F=8$ bits case.

\section{CONCLUSION}

In this paper we have studied different user selection criteria with quantized CDI and CQI feedback in order to increase the total throughput for a moderate number of users per cell. We have shown that, when the codebook is designed according to the local regions, the quantized version of the proposed criterion $\mathcal{T}_{3}$ outperforms the norm-only criterion $\mathcal{T}_{1}$. Furthermore we have shown that the performances are independent of quantized CQI whatever the number of feedback bits. Finally we point out that for the systems with thresholds at the receiver side, only CDI information is important at the transmitter to maximize the sum-rate performance.

\section{REFERENCES}

[1] M. Slim Alouini D. Gesbert, "How much feedback is multi-user diversity really worth?," In Proceedings of IEEE Intern. Conf. On Communications (ICC), 2004.

[2] T. Yoo and A. Goldsmith, "On the optimality of multiantenna broadcast scheduling using zero-forcing beamforming," IEEE Journal on Sel. Areas in Commun. (JSAC), vol. 24, no. 3, pp. 528-541, Mars 2006.

[3] E. Erkip B. Aazhang K. K. Mukkavilli, A. Sabharwal, "On beamforming with finite rate feedback in multiple antenna systems," IEEE Trans. on Information Theory, vol. 49, pp. 2562-79, Oct 2003.
[4] B. Hassibi M. Sharif, "On the capacity of mimo broadcast channels with partial side information," IEEE Trans. on Information Theory, vol. 51, pp. 506-522, Sept 2005.

[5] J. Andrews K. Huang, R. W. Heath, "Multi-user aware limited feedback for mimo systems," Subbmitted to IEEE Trans. on on Signal Processing, Jan 2007.

[6] M. D. Trott G. W. Wornell A. Narula, M. J. Lopez, "Efficient use of side information in multiple antenna data transmission over fading channels," IEEE Journal on selected areas in communications, pp. 1423-1436, Oct 1998.

[7] Jr. A. M. Sayeed V. Raghavan, R. W. Heath, "Systematic codebook designs for quantized beamforming in correlated mimo channels," IEEE Journal on selected areas in communications, vol. 25, pp. 1298-1310, Sept 1998. 